\title{
Cutaneous Angioneurofibroma: A New Histopathological Variant of Neurofibroma
}

\author{
Nikolina Saxer-Sekulic Gürkan Kaya \\ Dermatopathology Unit, Department of Dermatology, University Hospital of Geneva, \\ Geneva, Switzerland
}

Key Words

Angioneurofibroma $\cdot$ Neurofibroma Vascular endothelial growth factor

\section{Abstract}

Cutaneous neurofibromas are benign dermal tumors composed of spindle cells in a myxoid stroma containing numerous blood vessels. Here we describe 6 cases of solitary cutaneous neurofibroma showing unusually higher density of blood vessels in the stroma when compared to that of classical neurofibromas. We propose this lesion to be a new histopathological variant of neurofibroma and name it angioneurofibroma.

(c) 2014 S. Karger AG, Basel

\section{Background}

Neurofibromas are nonencapsulated, loosely textured dermal tumors composed of thin fascicles of cells with a spindle-shaped nucleus and scant cytoplasm [1]. The matrix is rich in mucin or sclerosing and hyalinized [2]. Blood vessels are increased in number in the stroma when compared to the normal skin, despite their white color in gross appearance [3]. Here we describe 6 cases of solitary cutaneous neurofibroma where the number of blood vessels in the stroma is unusually higher than that of classic neurofibromas. 
Fig. 1. Angioneurofibroma [hematoxylin-eosin; original magnification: $\times 5$ (a) and $\times 20$ (c)] and classical neurofibroma [hematoxylin-eosin; original magnification: $\times 5$ (b) and $\times 20$ (d)].

Saxer-Sekulic and Kaya: Cutaneous Angioneurofibroma: A New Histopathological Variant of Neurofibroma

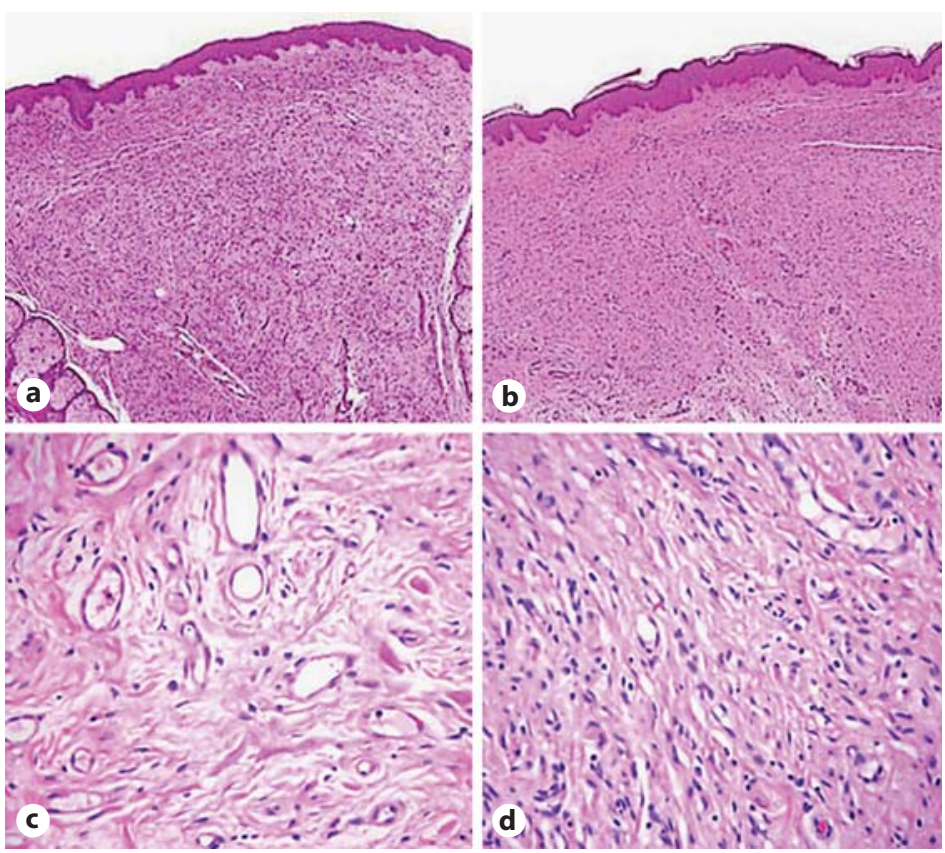

\section{Methods}

Biopsy material obtained from 6 patients with solitary cutaneous neurofibromas diagnosed as 'neurofibroma with a densely vascularized background' was reviewed and the number of blood vessels within the lesions was compared with 5 cases of solitary cutaneous neurofibroma showing a classical vascularized stroma.

The biopsy material was fixed in formalin, embedded in paraffin, cut at $5 \mu \mathrm{m}$ and stained with hematoxylin-eosin, and immunohistochemistry was performed for S-100, CD34 and D2-40 according to standard procedures. We counted the number of blood vessels of each lesion per 10 high-power fields $(\times 40)$ on anti-CD34-stained sections. Statistical analysis was performed by using an unpaired Student $t$ test.

\section{Results}

On histological examination, the well-known pattern of neurofibromas with an intact epidermis overlying a relatively ill-defined dermal proliferation of S-100-positive spindle cells with undulated nuclei in a collagenous background was observed in all of the cases (fig. 1). No atypia, mitosis or infiltrating pattern was observed. In the majority of the vessels, the endothelial cells showed a positive reaction pattern for CD34 (fig. 2c-d). There were also some vessels which were positive for D2-40, indicating their lymphatic origin (data not shown). The number of blood vessels was on average 50.7 ( \pm 15.4$)$ per 10 high-power fields $(\times 40)$ in the cases of 'neurofibroma with a densely vascularized background' whereas the average number of blood vessels counted was only $23.4( \pm 7.2)$ in the classical neurofibromas ( $\mathrm{p}<0.005$; unpaired Student t test; table 1; fig. 3). 
Fig. 2. S-100 immunostaining in angioneurofibroma (a; original magnification: $\times 20$ ) and classical neurofibroma (b; original magnification: $\times 20$ ); CD34 immunostaining in angioneurofibroma (c; original magnification: $\times 20)$ and classical neurofibroma (d; original magnification: $\times 20$ ).

Saxer-Sekulic and Kaya: Cutaneous Angioneurofibroma: A New Histopathological Variant of Neurofibroma
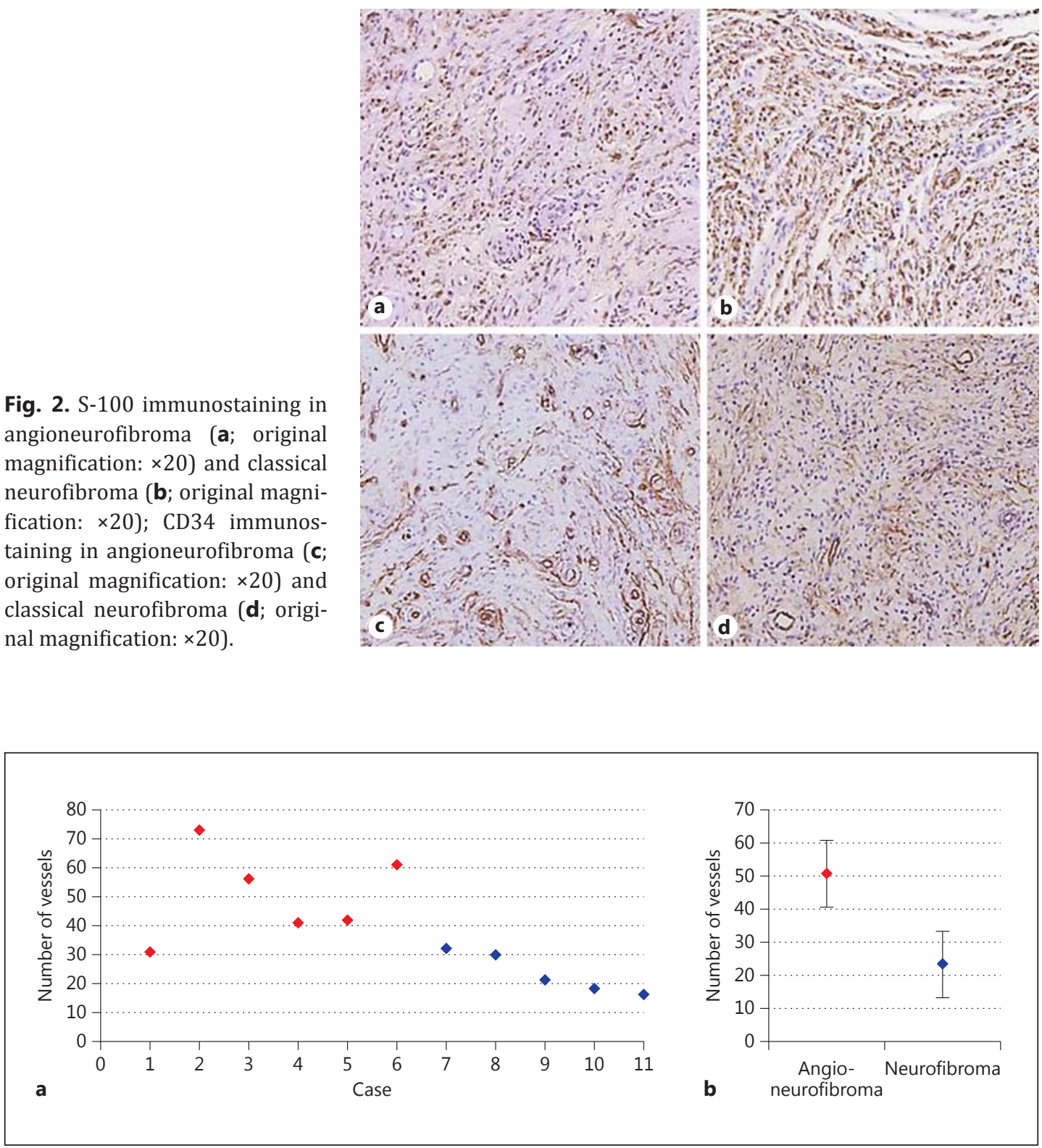

Fig. 3. Number of vessels in the cases of angioneurofibroma (1-6, red diamonds) compared with that of the cases of classical neurofibroma (7-11, blue diamonds) (a); standard deviation of the number of vessels in 6 cases of angioneurofibroma compared with 5 cases of classical neurofibromas (b) ( $p<0.005$; unpaired Student t test).

Table 1. Number of vessels in the cases of angioneurofibroma (1-6) and the cases of classical neurofibroma (7-11)

\begin{tabular}{llllllllllll}
\hline Case & 1 & 2 & 3 & 4 & 5 & 6 & 7 & 8 & 9 & 10 & 11 \\
\hline Number of vessels & 31 & 73 & 56 & 41 & 42 & 61 & 32 & 30 & 21 & 18 & 16 \\
\hline
\end{tabular}




\section{Discussion}

Neurofibromas are lesions characterized by delicate, in most cases only single cell-thick fascicles of cells with a spindled nucleus and scant cytoplasm [4], interpreted as Schwann cells on electron microscopy, and usually positive for S-100 protein [5]. The overlying epidermis is intact and separated from the lesion by a so-called dermal grenz zone. In some cases, an infiltrative pattern into the subcutis has been observed [1], and a vascularized stroma, sometimes abundant, has been documented [3]. In our cases, we were able to describe an unusually high vascular density compared not only with normal skin, but also with classical neurofibromas. In a series of neurofibromas from patients with neurofibromatosis-1 and sporadic neurofibromas, high vascular density associated with increased vascular endothelial growth factor immunoreactivity was shown [3]. This observation led to the suggestion that neurofibromas might have an angiogenic potential [3].

Megahed [1] described 10 histopathological variants of neurofibroma: classic, cellular, myxoid, hyalinized, epithelioid, plexiform, diffuse, pigmented, granular cell, and pacinian. Subsequently, some other variants such as dendritic cell neurofibroma with pseudorosettes [6] and lipomatous neurofibroma [7] have been reported. The histopathological pattern of the solitary cutaneous neurofibroma cases with a higher vascular density, which we describe here, is very particular. We therefore propose this lesion to be a new histopathological variant of neurofibroma and name it angioneurofibroma. Angioneurofibromas should be considered as a part of the morphological spectrum of neurofibromas. These lesions can be mixed histopathologically with vascular tumors. The gross appearance of angioneurofibromas should be compared to that of classical neurofibromas for any macroscopic difference. This feature might help to correctly interpret a highly vascularized lesion showing a histopathological pattern and immunohistochemical phenotype of neurofibroma, and to design new treatment strategies including angiogenesis inhibitors for these tumors.

\section{References}

1 Megahed M: Histopathological variants of neurofibroma. A study of 114 lesions. Am J Dermatopathol 1994; 16:486-495.

2 Shek TWH: Sclerosing neurofibroma. Histopathology 2000;36:377-378.

-3 Arbiser JL, Flynn E, Barnhill RL: Analysis of vascularity of human neurofibromas. J Am Acad Dermatol 1998; 38:950-954.

4 Reed RJ, Fine RM, Meltzer HD: Palisaded, encapsulated neuromas of the skin. Arch Dermatol 1972;106:865870.

5 Waggener JD: Ultrastructure of benign peripheral nerve sheath tumors. Cancer 1966;19:699-709.

6 Michal M, Fanburg-Smith JC, Mentzel T, Kutzner H, Requena L, Zamecnik M, Miettinen M: Dendritic cell neurofibroma with pseudorosettes: a report of 18 cases of a distinct and hitherto unrecognized neurofibroma variant. Am J Surg Pathol 2001;25:587-594.

7 Val-Bernal JF, de sa Dehesa J, Garijo MF, Val D: Cutaneous lipomatous neurofibroma. Am J Dermatopathol 2002;24:246-250. 